\title{
Improved Clustering Protocol to Balanced Energy Consumption in Heterogeneous Wireless Sensor Network
}

\author{
Santosh Vishnu Purkar*, Rajkumar S. Deshpande \\ Department of Electronics and Telecommunication, Savitribai Phule Pune University, India
}

Received January 15, 2019; Revised December 18, 2019; Accepted December 24, 2019

Copyright $@ 2019$ by authors, all rights reserved. Authors agree that this article remains permanently open access under the terms of the Creative Commons Attribution License 4.0 International License

\begin{abstract}
Wireless Sensor Network (WSN) Scheme is a cost-effective approach to monitor the event remotely. However, the activity of WSN is totally dependent on the energy resource available with each and every node of the network. Thus to prolong the activity period of WSN, it is necessary to utilize the available energy systematically. This aim is well satisfied by the design of hierarchical clustering protocol. This article highlights the mechanism to balance the energy consumption (load balancing) inside WSN. Proposed protocol design follows energy aware heterogeneous network model and modified scheme of cluster head $(\mathrm{CH})$ selection. Secondly, maintain the record for nodes utilized in the role of $\mathrm{CH}$ and its capability. Proposed approach explores energy utilization by rotating $\mathrm{CH}$ selection, and reduces the internal overheads in network management. Through this approach, we are able to improve the energy efficiency of WSN by clustering multilevel nodes. In this design, $\mathrm{CH}$ is selected based on node energy and relative distance attributes. Proposed protocol is simulated and validated with reference to energy based performance parameters. It's been noted that proposed implementation outperforms the existing protocols in terms of stability period, residual energy, and lifetime of the network.
\end{abstract}

Keywords HWSN, Propagation, Node Index, Multi-hop

\section{Introduction}

Wireless sensor network is an effective approach to monitor the interesting event remotely [1]. There are different approaches proposed by researchers in the form of time driven, event driven and query driven approach [2-3]. But, all are working properly if sensor nodes are alive. Thus, it is necessary to utilize the available node energy effectively in collecting information. There are different approaches proposed by the designers to enhance the energy efficiency of WSN [1-3]. Energy efficiency of the WSN can be improved by adding heterogeneity nodes inside the network. Secondly, by assigning specific role to the sensor nodes based on their capability and position [1]. In spite of the above option by reducing overloading of the particular node during communication will be one of the possible option to enhance the energy efficiency of the deployed network. This indirectly improves the lifetime of each individual and finally the life of the network. This further enhances energy remains with the network. Main outcome of this is lifetime get extend to a better level. To satisfy this need best possible option is to follow design based on clustering approach with the accountability of varied energy nodes. As normally nodes utilize with equal energy level (homogeneous WSN), but at run time there is variation in energy level available with the nodes utilized in network. This unequal energy utilization inside the network observed as a network with different energy level nodes. Thus, design based on clustering approach with Heterogeneous WSN (HWSN) is found to be the best for handling real life situation. This motivates us to design the clustering protocol with HWSN to balance energy over the network. This hybrid design approach with HWSN enhances the energy utilization over the network to a better level as compared to existing design. Application areas of WSN are in surveillance, intelligent traffic system, pollution control and in habitat monitoring as presented in [1-3]. Latest application area improves the publicity of WSN to high level is Industry Internet of Things (IIOT or Industry Revolution fourth generation or I4.0).

The prime objective of this design is to achieve load balancing over the network for long span of time. In this paper, we present a best possible approach to select $\mathrm{CH}$, such that selected $\mathrm{CH}$ use minimum energy to communicate with BS. Selection of $\mathrm{CH}$ is very important in cluster design, as compared to other control activity. Thus 
it initiates minimization of energy consumption with deployed network. We try to rotate the role of $\mathrm{CH}$ after each cluster round such that all the available nodes from the network get equal chance for the role of $\mathrm{CH}$. This enhances the load balancing over the network [1-3].

Organization of the paper is as presented in following subsections. Literature survey based on published articles is highlighted in section 2. Highlights of proposed system model is presented in section 3. An algorithm detail is given in section 4. Brief results discussion is given in section 5 . Conclusion of the proposed implementation is written in final subsection 6 .

\section{Literature Survey}

Main aim of this proposed design is to utilize the WSN platform to monitor the event in cost effective manner. As sensor nodes are energy constraints elements and thus WSN in remote event monitoring is constrained with node energy, it is highly impossible in most of the case to recharge the available node remotely, thus network lifetime is affected badly. Thus, to extend the service of WSN or Heterogeneous WSN it is necessary to utilize the available energy smartly. This aim motivates the researchers to design energy efficient scheme for WSN such that lifetime of the network can prolong. Cluster based routing scheme achieves the same to a better level than other alternatives. Some of the outcomes of this approach are enhanced load balancing, by offering chance to each node to participate in the role of $\mathrm{CH}$. Some of the improvements with clustering approach are fault tolerant, reduced internal overheads and avoid collision. Clustering schemes are classified as probabilistic and non-probabilistic. Alternatively Centralized, Distributed and Adaptive clustering. Scheme selection is based on applications requirement. Some of the examples of these schemes are LEACH [4], SEP [5], DEEC [6].

\subsection{LEACH Protocol}

To enhance the load balancing to improve the energy efficiency of the network author proposes Low-Energy Adaptive Clustering Hierarchy (LEACH) protocol in [4]. This innovative approach of $\mathrm{CH}$ rotation improves load balancing and hence lifetime of each individual by offering the chance to participate in data communication. Unfortunately, $\mathrm{CH}$ selection is random (probabilistic approach). As $\mathrm{CH}$ selection is without considering node energy or network energy and without relative distance selected $\mathrm{CH}$ seems to be in competent. This result in arbitrary cluster formation may result in quick death of selected $\mathrm{CH}$. Thus results in black hole in the network. This may divide the network into different sections. However, LEACH is direct communication scheme with $\mathrm{CH}$ or with base station. Main problem is selected $\mathrm{CH}$ not guaranteed off the boundary. This scheme treats all nodes as equal capability nodes during network lifetime, which is less feasible in real time environment. Thus, it is less suitable design scheme in remote event monitoring [4]

\subsection{SEP Protocol}

To resolve the issues of LEACH and to justify the real life application need. Author of Stable Election Protocol (SEP) proposes clustering protocol based on heterogenite nodes in WSN [5]. Here, CH selection is based on initial energy of the node. In this design the author adds high energy nodes in normal node WSN (nodes of equal capability or equal energy) to form heterogeneous WSN. Added nodes are termed as advanced nodes. This design tries to offer justice to HWSN network model by shifting the $\mathrm{CH}$ selection based on energy. SEP design prolongs the stability and lifetime of the network. Epoch is adjusted such that high energy nodes are utilized higher than lower energy nodes in the role of $\mathrm{CH}$. However, this result in high utilization of advanced node compared to low energy nodes, and this result in early death of the node. To minimize the load of or to reduce balanced the load of advanced node author of Enhanced SEP increases the heterogeneity level to three in the form of [7]. But, it has the same problem as SEP and less aware of network energy.

\subsection{DEEC Protocol}

The issue of network energy is other component need to be considered while selecting $\mathrm{CH}$. This improves energy efficiency of the network to a better level than the previous approach. To support the same, an innovative approach of $\mathrm{CH}$ selection is based on node and the network energy is presented by the author of Distributed Energy Efficient Clustering (DEEC) in [6]. This approach follows the varied epoch period as per node energy. These two types of node protocols are facing the problem of internal computation and early death of advanced nodes. Dilip Kumar et. al extends the work of SEP and DEEC in the form of Energy Efficient Heterogeneous Clusters (EEHC) algorithm in [8]. But, node deployment needs to be systematic.

Hence, we proposed the clustering approach with three type nodes namely normal, advanced and super. This designed scheme offers better performance as compared to former design approach in terms of stability; lifetime and energy remaining in the network.

\section{System Model}

In this scheme, we use the radio model as utilized in [9]. Proposed network model is based on 3 type nodes. We use $\mathrm{n}=200$ randomly deployed nodes in the form of $M \times M$ supervisory field. All nodes are immobile after deployment. BS is placed at the center of network field. Network field is initially divided into four sections. Communication 
channels are assumed to be bidirectional [9].

\subsection{Energy Consumption Model}

Energy consumption model is divided in two sections as transmitting and receiving section. Transmitting section consists of amplifying circuits, radio electronics and processing circuit, whereas for receiver radio electronics and processing electronics. These two entities are separated by a distance (safe distance or threshold distance) $\mathbf{d}_{\mathbf{0}}[11]$. Energy consumption attributes are $E_{\varepsilon f s}$ the energy of propagation over free space by satisfying the condition as $\mathrm{d}<<\mathrm{d}_{0}$, and in multipath propagation over the distance $\mathrm{d}>>\mathrm{d}_{0}$ as $\mathrm{E}_{\mathrm{mp}}$. Here, $\mathrm{d}$ is the distance over which communication to be expected. Data aggregation model utilized for L-byte message is same as given in [4-5, 8-9].

\subsection{HWSN Model}

In this network model, all nodes are energy heterogeneous with varied energy levels. As first type nodes termed as normal, with energy level as $\mathrm{E}_{0}$. Second type nodes are advanced nodes with fractional percentage factor $\mathbf{m}$ with total $\mathrm{n}$ nodes. These nodes have a time more energy than the normal nodes. Thus, energy level is $\mathrm{E}_{0}(1+\mathrm{a})$. Super nodes the third type nodes with percentage fraction value $\mathbf{m}_{\mathbf{0}}$ with respect to $\mathbf{n}$ total nodes, and $\mathbf{b}$ times more energy than first normal nodes. Thus, energy level is $\mathrm{E}_{0}(1+\mathrm{b})$. Thus final compositions of nodes populations are $n\left(1-m-m_{0}\right), n_{0}$, and $n m$. Energy levels of three type nodes are as $n\left(1-m-m_{0}\right) E 0, n E_{0} m(1+a)$ and $n_{0} E_{0}(1+b)$. Thus, total value of energy level composition for network is presented as; $\mathrm{nE}_{0}\left(1+\mathrm{m}\left(\mathrm{a}+\mathrm{m}_{0} \mathrm{~b}\right)\right)$ [8-9].

\subsection{Proposed Protocol}

In this design, we proposed non-probabilistic designed approach with three type node network models. Proposed design is based on new node profile parameter as Node Quality Index (NQI). This profile parameter is nothing but the regression of residual energy, initial energy and relative distance with the base station. We design this proposed scheme in two stages as proposed design by keeping fixed epoch period and second stage with varied epoch period by following the same algorithm stages. We update value of NQI at the end of every cluster cycle. Inspite of the above considerations, we also utilize the boundary condition in $\mathrm{CH}$ selection $\mathrm{CH}$ additively.

\subsubsection{Proposed Algorithm}

In primary stage, all nodes send their respective attributes to BS. Further, BS calculates node index of every node, and performs averaging and filtering.

\section{A. First stage of implementation}

The nodes, those have better value of node index than others, are selected as temporary $\mathrm{CH}$ for that region. Finally, the nodes who have better node index than temporary nodes are selected as final $\mathrm{CH}$ for that corresponding round. This node on behalf of network communicates all aggregated data from the network to BS. Here, all deployed nodes are location aware, hence may consume small amount of energy during operation. Nodes always check the distance with nearby node before sending the data. If this distance is greater than the distance with BS, then sent all collected data to BS directly. Alternatively, can be sent to neighboring node. All other activities of $\mathrm{CH}$ are same as per the duty of $\mathrm{CH}$.

\section{B. Second stage of implementation}

In second stage, modified scheme of $\mathrm{CH}$ selection based on energy aware epoch to finalize proper nodes for the role of $\mathrm{CH}$. Nodes corresponding node index is also checked before finalizing the $\mathrm{CH}$. Nodes finalized for the role of $\mathrm{CH}$ and has low index value, strictly avoided. To improve the load balancing more effectively LEACH strategy is utilized for a small number of rounds at the start of algorithm.

\section{Simulation Results}

This section highlights the performance parameters utilized to validate the proposed implementation as given in [9]. This section also introduced radio parameter details used in simulating proposed protocol.

List of performance parameters is presented as follows:

\subsection{Stability Period}

The time period in the form of cluster round before the death of very first node from the network during operation is termed as stability period.

\subsection{Lifetime}

The time elapsed in the form of cluster rounds before the death of last available node from working network is termed as lifetime of network under supervision.

\subsection{Number of Alive Nodes per cluster round}

Number of Alive nodes per cluster round presents during event monitoring cycle depicts aliveness of nodes over a cluster round.

\subsection{Energy remain in the network per cluster round}

Energy remain in the network per cluster round is the residual energy of the network over a cluster round.

\subsection{Results discussion}

Radio parameters set utilized in simulating proposed design is presented in the form of table 1 as shown below in the form of simulation parameters.

Simulation of proposed implementation is completed in two stages with respect to LEACH, SEP and DEEC protocol. Here, proposed HWSN model utilize three different types of nodes. In this simulation, we used the $10 \%$ super nodes and $20 \%$ advanced nodes of the total available nodes. 
Table 1. Simulation Parameters

\begin{tabular}{|c|c|c|}
\hline \multicolumn{2}{|c|}{ Simulation parameter } \\
\hline Parameter symbol & Name & $200 \mathrm{mX} 200 \mathrm{~m}$ \\
\hline- & Network area & 200 \\
\hline $\mathrm{N}$ & Number of nodes & $0.5-1.5 \mathrm{~J}$ \\
\hline $\mathrm{E}_{0}$ & Initial energy of nodes & $4000 \mathrm{bytes}$ \\
\hline $\mathrm{L}$ & Data packet size & $50 \mathrm{~nJ} / \mathrm{bit}$ \\
\hline $\mathrm{E}_{\text {elec }}$ & Radio electronics energy & $10 \mathrm{pJ} / \mathrm{bit} / \mathrm{m} 2$ \\
\hline $\mathrm{E}_{\varepsilon \mathrm{fs}}$ & Free space energy & $0.0013 \mathrm{pJ} / \mathrm{bit} / \mathrm{m} 4$ \\
\hline $\mathrm{E}_{\mathrm{mp}}$ & Amplification energy & $5 \mathrm{~nJ} / \mathrm{bit} / \mathrm{signal}$ \\
\hline $\mathrm{E}_{\mathrm{DA}}$ & Data aggregation energy & $87-87.7 \mathrm{~m}$ \\
\hline $\mathrm{d}_{0}$ & Threshold distance & $100 \mathrm{X} 100$ \\
\hline- & Sink node & \\
\hline
\end{tabular}

Here, simulation is completed with reference to 200 nodes. First stage comprises fixed epoch period in $\mathrm{CH}$ selection. Due to the use of node initial energy, residual energy and hop count in calculating node index. Averaging of node index decide the most promising node for the role of $\mathrm{CH}$. Selected $\mathrm{CH}$ must satisfy the boundary condition such that selected $\mathrm{CH}$ is off the network boundary. Nodes that have highest node index value and less time utilized in $\mathrm{CH}$ role is selected as final $\mathrm{CH}$ node on behalf of the network. Final $\mathrm{CH}$ collect the information from regional $\mathrm{CH}$ and communicate it to BS. After completing cluster cycle again, the process of $\mathrm{CH}$ selection is started at the end of cluster round. Thus available node energy is utilized faithfully during network operation. Hence node aliveness period gets prolonged to longer time period. Fig. 1 presents alive nodes versus number of cluster rounds and Fig. 2 presents dead nodes Vs number of cluster round. This presents the reverse condition of alive nodes per cluster round. In observed simulation, we found that LEACH protocol supports load balancing, but with probability based approach. Thus, $\mathrm{CH}$ selection is less faithful as compared to other proposed cluster design. This may result in arbitrary cluster size inside the network. Hence, this affects the $\mathrm{CH}$ energy level and may result in death of the node. LEACH's CH selection is less faithful as compared to SEP and DEEC. But, with SEP there is reference of energy utilized in selecting $\mathrm{CH}$ and based on the epoch period, this may result in advance node selection for the role of $\mathrm{CH}$. Thus, it may result in death of the node. But, improved performance as compared to LEACH. In DEEC, network maintains global information in calculating lifetime of the network. But $\mathrm{CH}$ selection is based on residual energy of the node to average energy of the network. This also results death of advanced node same as SEP. Proposed two stage approach of load balancing offer best node degree, better intra-connectivity inside the network. Thus, hybrid approach of direct and Multi-hop communication enhances the energy efficiency of the network as compared to LEACH, SEP and DEEC as depicted in Fig. 3. 


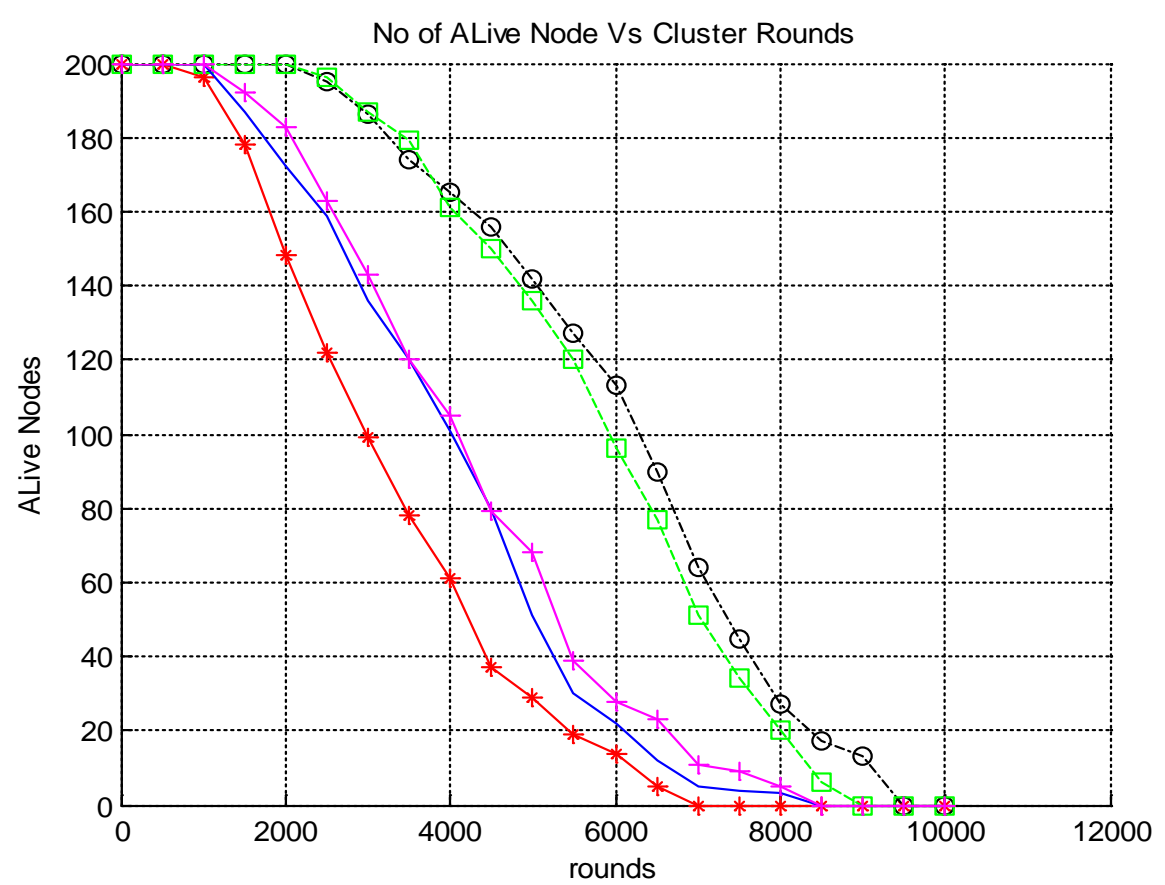

Figure. 1. No. of Alive nodes versus cluster rounds.

In Fig. 1, 2 and 3, colored graph with green and black colored (diamond and circle respectively) presents our proposed design with two stage mechanisms, with circle and diamond shape. Other colours, red, blue and pink, are utilized for the plot of LEACH, SEP and DEEC protocol. We follow *, simple plane line and $\mathbf{x}$ sign on the plot.

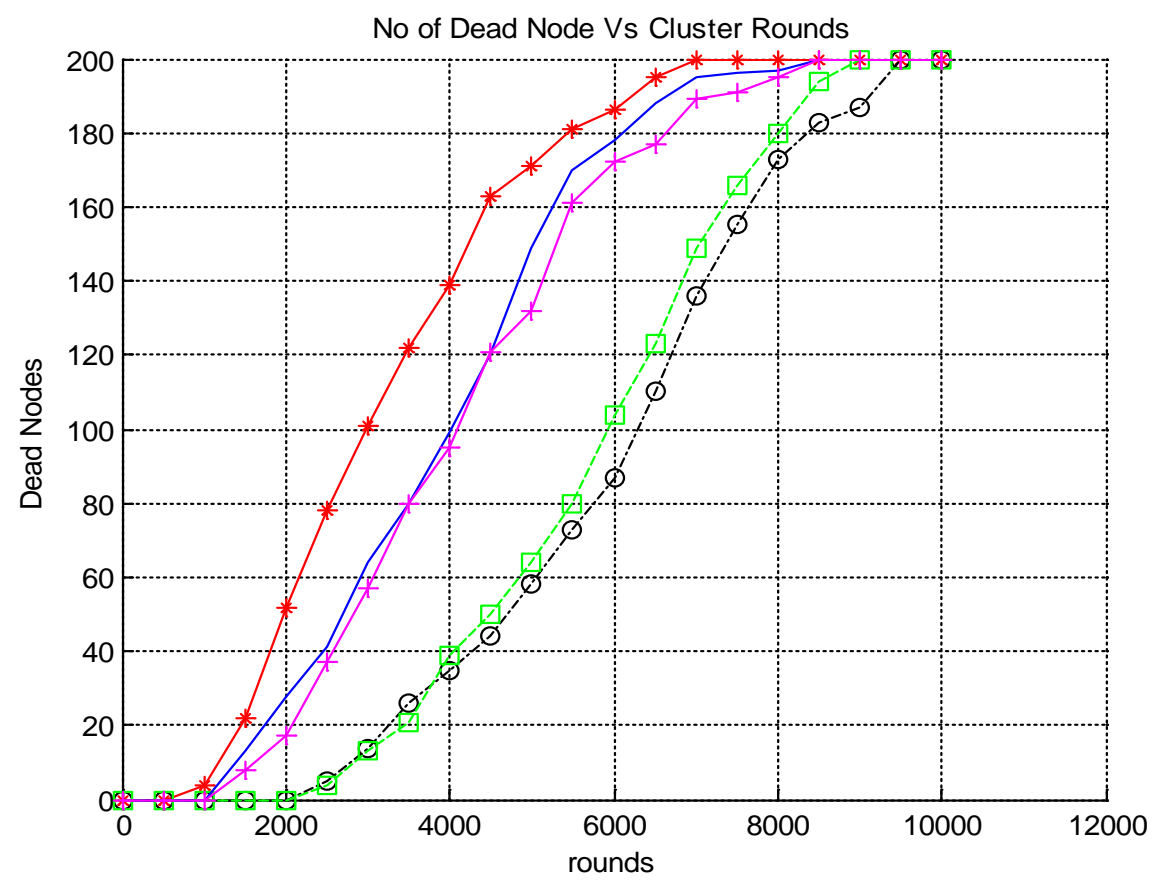

Figure 2. No .of Dead nodes versus cluster rounds. 


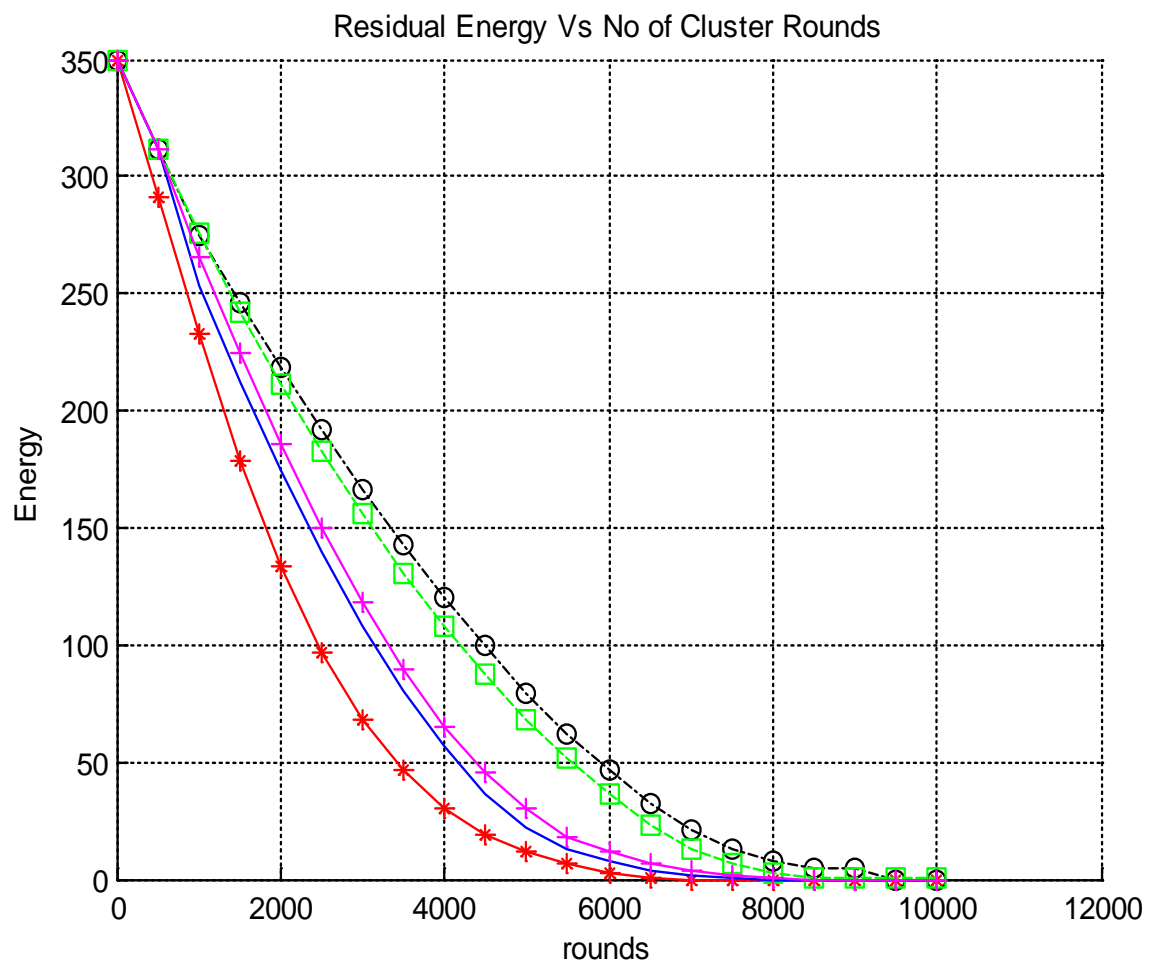

Figure 3. Energy remains in the network Vs cluster rounds.

Following, Fig. 4 shows the performance parameter recorded from simulation environment in the form of bar chart.

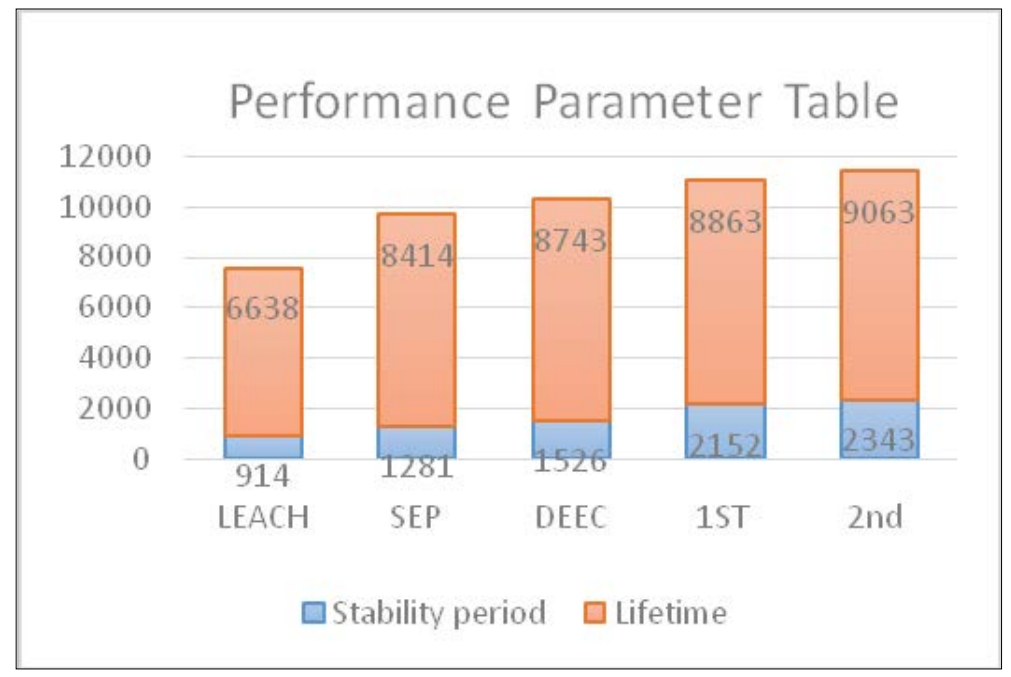

Figure 4 Performance Parameter Chart 


\section{Conclusions}

This paper presents an approach of efficient utilization of residual energy to prolong the performance parameters of the HWSN. This mechanism achieves multiple benefits such as less internal overheads, better network management and limited cluster size. Additional benefits offered by this design scheme are better $\mathrm{CH}$ selection, improves load balancing and prolonged lifetime. This improvement in energy utilization signifies that this designed hybrid approach is more energy conscious than available existing $\mathrm{CH}$ (clustering protocol) selection protocol. It is being identified that designed approach works better in different epoch environments as compared to existing protocols. In future, we try to explore our work towards cluster head improvement and throughput improvement with genetic algorithm scheme.

\section{REFERENCES}

[1] Sheikhpour R, Jabbehdari S, Khadem-Zadeh A (2011) Comparison of energy efficient clustering protocols in Heterogeneous wireless sensor networks. International Journal of Advanced Science and Technology, pp. 27-40.

[2] Abbasi AA, Younis M (2007) A survey on clustering algorithms for wireless sensor networks. Science Direct Computer Communications, pp. 2826-2841.

[3] Purkar SV, Deshpande RS (2017) A Review on Energy Efficient Clustering Protocols of Heterogeneous Wireless Sensor Network. International Journal of Engineering and Technology: 9(3), pp.2514-2527.

[4] Heinzelman WR, Chandrakasan A, Balakrishnan H (2000) Energy-Efficient Communication Protocol for Wireless Micro-sensor Networks. In Proceedings of the 33rd Annual Hawaii International Conference on System Sciences, Maui, HI, USA pp. 10-19.

[5] Smaragdakis G, Matta I, Bestavros A (2004) SEP: A Stable Election Protocol for clustered heterogeneous wireless sensor network. Second International Workshop on Sensor and Adhoc Network Protocols and Applications, pp.1-6.

[6] G, Qing L, Zhu Q, Wang M (2006) Design of a distributed energy-efficient clustering algorithm for heterogeneous wireless sensor network. ELSEVIER, Computer Commu. Vol.29, pp 2230- 2237.

[7] Min X, Wei-ren S, Chang-jiang J, Ying Z (2010) Energy efficient clustering algorithm for maximizing lifetime of wireless sensor networks. Int. J. Electron. Commun. Vol. 64, pp. 289-298.

[8] Kumar D, Aseri TC, Patel RB (2009) EEHC: Energy efficient heterogeneous cluster scheme for wireless sensor networks. Elsevier computer communication, pp.662-667.

[9] Purkar SV, Deshpande RS (2018) Energy Efficient Clustering Protocol to Enhance Performance of Heterogeneous Wireless Sensor Network: EECPEP-HWSN.
Journal of Computer Networks and Communication: 2018 (2078627), pp.1-12. 